\title{
Desafío epistemológico de los conocimientos educativos indígena y escolar para una educación intercultural'
}

\section{Resumen}

\author{
Esteban Saavedra ${ }^{2}$ \\ ORCID: 0000-0002-9220-4320 \\ Daniel Quilaqueo ${ }^{2}$ \\ ORCID: 0000-0002-5143-4920
}

El propósito de este trabajo es comprender y explicar la tensión epistemológica entre los conocimientos educativos indígenas con la monoculturalidad eurocéntrica de los sistemas educativos nacionales, desde la complejidad que implica la educación escolar en los contextos de los pueblos indígenas de América. Puesto que la justificación ha sido la colonización y su integración a los Estados nacionales, negando las epistemes de sus conocimientos educativos en la educación escolar. Frente a esto, proponemos una crítica epistemológica a la construcción de conocimientos interculturales propuesta por los Estados, apoyándonos en el pluralismo epistemológico. Específicamente, analizamos los casos del proyecto Milpas educativas de México, la acción educativa kimeltuwün en la Araucanía, Chile y la política educativa en el estado de Roraima, Brasil. Se considera el contexto político que norma la educación intercultural sobre las experiencias de Educación Intercultural Bilingüe de los estados nacionales y las recomendaciones del Convenio 169, de la Organización Internacional del Trabajo. En conclusión, planteamos una educación intercultural tomando en cuenta la tensión epistemológica entre las epistemes indígenas y escolar. Asimismo, planteamos la necesidad de construir una educación intercultural, contextualizada a cada pueblo indígena, en el marco de sus relaciones interétnicas con los no indígenas mediante un diálogo desde la perspectiva del pluralismo epistemológico.

\section{Palabras clave}

Educación intercultural - Pluralismo cultural - Relaciones interétnicas - Epistemología.

1- Esta investigación ha sido financiada por la Comisión Nacional de Investigación en Ciencia y Tecnología (CONICYT - Chile) a través del proyecto FONDECYT Regular N¹181314.

2- Universidad Católica de Temuco, Temuco, Chile. Contactos: esaavedra@educa.uct.cl; dquilaq@uct.cl. 


\section{The epistemological challenge of indigenous and school educational knowledge in intercultural education}

\section{Abstract}

The object of this work is to understand and explain the epistemological tension between indigenous educational knowledge and the Eurocentric monoculturality of national education systems, from the complexity implied by school education in the contexts of the indigenous peoples of America. The justification for this style of education has been the colonisation of indigenous peoples and their integration into national states, denying the epistemes of their own educational knowledge in school education. In this scenario, we propose an epistemological critique of the construction of intercultural knowledge as proposed by states, based on epistemological pluralism. Specifically, we analyse the cases of the Milpas Educativas project in Mexico, the educational action called kimeltuwün in the Araucania Region of Chile, and the education policy of the State of Roraima, Brazil. We consider the political context that regulates intercultural education in the Bilingual Intercultural Education experiments of national states and the recommendations of Convention No. 169 of the International Labour Organisation. In conclusion, we propose a form of intercultural education that takes into account the epistemological tension between the indigenous and school epistemes. We also propose the need to construct a form of intercultural education adapted to the context of each indigenous people, in the framework of their interethnic relations with non-indigenous societies, through dialogue from the perspective of epistemological pluralism.

\section{Keywords}

Intercultural education - Cultural pluralism - Interethnic relations - Epistemology.

\section{Introducción}

En el presente artículo se realiza una crítica epistemológica a la construcción de conocimientos interculturales en las escuelas, desde las investigaciones de académicos latinoamericanos que estudian la incorporación de las epistemes indígenas al currículum escolar. En esta crítica subyace la idea propia de la educación indígena, que ha sido negada en las escuelas, y la predominancia de la educación escolar monocultural utilizada como medio de colonización e integración de los pueblos indígenas a los estados nacionales. Para el análisis se consideran tres perspectivas de incorporación de conocimientos educativos indigenas en la educación escolar desde enfoques interculturales de tres casos: proyecto Milpas educativas de México (BERTELY, 2015; BERTELY; SARTORELLO; ARCOS, 2008); proyecto educativo en el estado de Roraima, Brasil (REPETTO, 2012, 2019; REPETTO; 
CARVALHO, 2015); y kimeltuwün o acción educativa mapuche, en la Araucanía, Chile (QUILAQUE0; QUINTRIQUE0, 2017).

Los tres casos señalados se han escogido debido a que aportan diferentes métodos y contextos para abordar el tema de conocimientos indígenas y escolar en la incorporación de contenidos de educación intercultural. El caso del proyecto Milpas educativas aporta conocimientos sobre un modelo educativo intercultural plurilingüe; en el caso del proyecto educativo en el estado de Roraima, en Brasil, se evidencian tensiones entre el estado con empresarios y comunidades indígenas; y la acción educativa kimeltuwün, muestra epistemes indígenas que se diferencian y en varios puntos se contrapone con la pedagogía impartida en las escuelas.

El análisis de los tres casos señalados busca reconocer los puntos comunes de la implementación de la educación intercultural entre los pueblos indígenas y las características epistémicas de la educación indígena. Para ello, recurrimos al pluralismo epistemológico de Olivé $(1999,2004,2009)$ como base que permite la reflexión crítica sobre las prácticas educativas en contextos indígenas. La propuesta de este trabajo es comprender y explicar la tensión epistemológica entre los conocimientos educativos indígenas con la monoculturalidad eurocéntrica de los sistemas educativos nacionales (BERTELY, 2014; REPETTO, 2012; WALSH, 2010). Asimismo, el enfoque intercultural, según Olivé (1999), permite reconocer que las visiones absolutistas o relativistas extremas no son las apropiadas para la construcción de conocimientos en contexto de escuelas con estudiantes de origen indígena. Lo que se busca, es el "[...] descentramiento, el descubrimiento del marco de referencia del otro, la negociación y la mediación” (COHEN-EMERIQUE, 2013, p. 2). Es decir, promover relaciones basadas en el respeto, que contrarreste la monoculturalidad y exclusión entre los distintos grupos culturales (GROSFOGUEL, 2016; SANTOS, 2012).

A partir de estos planteamientos, se observa que el problema en la construcción de educación intercultural en las escuelas se refleja en las relaciones asimétricas entre los pueblos indígenas y descendientes de europeos (BRIONES, 2013; OLIVÉ, 1999, 2004), puesto que, a pesar de las buenas intenciones, las relaciones interétnicas manifiestan la exclusión y discriminación en las instituciones sociales. Es lo que afecta particularmente a los pueblos indígenas de México, Brasil y Chile, donde se constatan las condiciones que han promovido el epistemicidio de los conocimientos indígenas (RIVERA CUSICANQUI, 2010; SANTOS, 2017). Al respecto, Schmelkes (2004) devela en sus estudios dos situaciones: 1) la existencia de asimetrías escolares que permiten que haya menos acceso a las escuelas por parte de las poblaciones indígenas; y 2) la más grave, que los indígenas aprenden menos en las escuelas porque no es contextualizada y no se les enseña lo que realmente necesitan conocer. Por consiguiente, consideramos que para contrarrestar el epistemicidio, el pluralismo epistemológico permite reconocer e incorporar conocimientos indígenas en la construcción de educación intercultural en contextos indígenas (OLIVE, 1999, 2004, 2009).

La propuesta de educación intercultural, desde el pluralismo epistemológico, permite la interacción y negociación entre distintos actores sociales para conseguir una formación de personas conscientes de las diferencias y capaces de trabajar en conjunto para co-construir una sociedad plural y más justa (WALSH, 2010). Asimismo, acepta distintas perspectivas que cambian las definiciones tradicionales de la realidad social en la construcción de 
conocimiento (BERGER; LUCKMANN, 1991), puesto que implica incorporar el contexto social y convivencia de personas con distintas culturas y formas de vivir. Al respecto, vemos como la educación intercultural permite la construcción de conocimiento entre saberes indígenas en conjunto con lo que se considera conocimiento universal basado en la deducción y la totalidad (ALCOREZA, 2014). El pluralismo epistemológico otorga criterios de validez que posibilitan la legitimación de los conocimientos tradicionales, en relación los conocimientos científicos (OLIVÉ, 2009). Por ejemplo, es lo que se observa en el Método Inductivo Intercultural (MII) propuesto por Gasché (2013), que promueve una educación intercultural basada en las conceptualizaciones de interculturalidad superando las relaciones de tolerancia de la multiculturalidad.

Este artículo considera como marco de referencia los conceptos de multiculturalidad e interculturalidad. Desde el concepto interculturalidad, según lo planteado por FornetBetancourt (2002), el solo hecho de definirlo supone un conflicto debido a que la necesidad de una definición universal es propia del eurocentrismo positivista, cuyas definiciones suelen objetivar lo definido y se articulan desde un área disciplinar. En tanto, la multiculturalidad educativa enfatiza el rescate y preservación de la cultura propia, igualar las oportunidades sin diferenciar su procedencia étnica, cultural o situación económica (BANKS, 1995). A esta definición, Sleeter (2018) adiciona la idea de un concepto más elevado que el abordaje del tema desde la didáctica y currículum, para esta autora también es un espacio de lucha en un mundo desigual.

Por su parte, la interculturalidad, dado su carácter de interacción y promoción de la convivencia entre personas o entidades de distintas culturas en un mismo lugar, propone que las interacciones sociales sean sin discriminación, con igualdad de derechos y en respeto por la diversidad (ROMER0, 2003). Es decir, propone favorecer la superación de la mera tolerancia cultural e ir por el camino de la convivencia social para el enriquecimiento de todos, en todo sentido, para desarrollar una transformación cultural (FORNETBETANCOURT, 2002). Este camino tiene el desafío de reivindicar las culturas dominadas que han sobrevivido al colonialismo y homogenización de las escuelas de los estados naciones (GUILHERME; DIETZ, 2015). Es decir, el reconocimiento de la diversidad cultural y el despertar de la conciencia pluricultural a nivel mundial a causa de la globalización, entre otras (SCHMELKES, 2004). Producto de este reconocimiento a la variedad de culturas, la producción científica en el área de educación intercultural ha ido en aumento desde los años noventa hasta la actualidad, abarcando diferentes ámbitos como la ética, política, epistemología y educación (FERRÃO CANDAU, 2010).

En síntesis, el foco de este ensayo es la episteme indígena y escolar en relación a la construcción de conocimiento intercultural en las escuelas, sin dejar fuera las luchas de los movimientos sociales indígenas y sus demandas por reconocimiento, derechos y transformación social (WALSH, 2010), puesto que han permitido el avance en la construcción de la educación intercultural en América Latina donde las relaciones de dominación social y política produjeron las asimetrías que aún siguen vigentes (BRIONES, 2013).

El texto se estructura de la siguiente forma: 1) evolución normativa de la educación intercultural en América Latina, en la que se describe el contexto político que norma la educación intercultural, con énfasis en la Educación intercultural Bilingüe y el Convenio 
169 de la Organización Internacional del Trabajo (OIT, 1989); 2) descripción y evidencia de tensiones sociales y políticas que viven los pueblos indígenas en el estado de Roraima, Brasil a partir de los estudios de Repetto (2012, 2019) y Repetto y Carvalho (2015); 3) descripción y explicación del proyecto Milpas educativas, de un grupo de investigadores de universidades mexicanas, los que implementan una didáctica de enseñanza con epistemes de pueblos indígenas mexicanos; 4) descripción y explicación de la episteme mapuche, de los pueblos mapuches chilenos ubicados en el centrosur de Chile, desde la acción educativa kimeltuwün; y 5) conclusión con una reflexión crítica sobre la construcción de conocimientos interculturales en América Latina.

\section{Evolución normativa de la educación intercultural en América Latina}

La normativa de la educación intercultural se ha puesto en las agendas de los gobiernos latinoamericanos debido a las reivindicaciones de distintos pueblos indígenas, que se movilizan por una educación escolar que considere sus culturas y educación propia, para poder coexistir dentro de una misma sociedad nacional. Esto refleja que la construcción de conocimientos interculturales en las escuelas no ha estado exenta de tensiones, debido a la "[...] identificación cultural, derecho y diferencia, autonomía y nación" (WALSH, 2010, p. 5).

La educación intercultural comienza a ser estudiada y tomada en cuenta a nivel político en América Latina desde los años 80. La política educativa de México ha sido pionera con propuestas en la Educación Intercultural Bilingüe (EIB) (WALSH, 2010). Según López y Küper, su implementación mejora el "[...] desarrollo cognoscitivo y afectivo de los educandos a los que hasta ahora se ha dirigido los indígenas" (1999, p. 72). Sin embargo, la EIB no ha estado exenta de críticas al ser catalogada como una actividad organizada desde arriba, por el Estado, sin la participación de los pueblos indígenas (WILLIAMSON, 2004).

De lo anterior, el hito importante que continúa con la construcción de una educación intercultural tiene relación con la firma del Convenio 169 de la OIT. Este instrumento jurídico internacional establece los principios y obligaciones de los Estados nacionales con los pueblos indígenas (COURTIS, 2009). Su ratificación, en la mayoría de los países latinoamericanos, fue inmediatamente posterior a su firma en la convención celebrada en Ginebra, Suiza en 1989. Por ejemplo, México lo hizo en 1990 y Brasil en 2002 (ASSIES, 2007), sin embargo, Chile se abstuvo de votar, lo que postergó la ratificación de este Convenio hasta el año 2007 (DONOSO, 2008).

El Convenio 169 establece los derechos de los pueblos indígenas tales como, decidir sus prioridades de desarrollo, conservar sus costumbres e instituciones, utilizar recursos naturales disponibles en sus territorios, participar en las políticas que les afecten directamente y reafirmar sus derechos en cultura, lengua y educación (DONOSO, 2008). Respecto a educación, este Convenio señala la igualdad de derechos, la contextualización abarcando la historia, conocimientos y técnicas propias de los pueblos indígenas y participación en la creación de los programas de estudio; entre otras. Sin embargo, este Convenio es una declaración que manifiesta las diferencias entre indígenas y occidentales, 
mostrando que no pertenecen a una misma sociedad, puesto que, “[...] resulta claramente que el universo de vida indigena es considerado diferente del universo de la sociedad nacional u occidental, y que los derechos especiales del Convenio son la consecuencia de esta diferencia” (GASCHÉ 2008, p. 12).

En síntesis, el ámbito normativo es importante para la construcción de una educación intercultural. No obstante, las instituciones encargadas por crear estos acuerdos, por aplicarlos en los países y responsabilizarse de ellos, a la vez son los responsables de la perpetuación de la dominación hegemónica de los pueblos indígenas, puesto que deslegitiman sus aspiraciones educativas en relación a la contextualización de la enseñanza y la no consideración de las epistemes que los pueblos indígenas han utilizado por siglos en la formación de hijos.

\section{Proyecto educativo intercultural en Roraima}

En el estado brasilero de Roraima, situado en la región norte de Brasil, viven pueblos indígenas que se clasifican en las siguientes familias lingüísticas: 1) de la lengua karibe encontramos los pueblos Makuxi, Taurepang, Ingarikó, Yekuana, Patamona, Sapará, WaiWai, Waimiri-Atroari; 2) de la lengua aruak están los Wapixama; y 3) de la lengua Yanomami se encuentra el pueblo con homónimo a la lengua (MANDULÃo et al., 2012; REPETTO, 2012).

Los pueblos indígenas presentes en Roraima muestran una complicación en relación a su idioma puesto que "[...] nem eles mesmos passaram a falar as línguas indígenas que a geração de pais e avós deliberadamente não ensinaram, como uma estratégia de invisibilização frente ao preconceito e a exclusão cultural que enfrentavam nas escolas e no contexto regional" (REPETTO, 2019, p. 81). Estas prácticas de protección contra la discriminación que han utilizado abuelos y padres ha contribuido a la homogenización cultural de los hijos para su integración a la sociedad brasilera.

Desde el ámbito normativo, estos pueblos se caracterizan por mantener diferentes propuestas educativas impulsadas desde el marco normativo de la política educativa indígena de Brasil (REPETTO, 2012). En ella, la propuesta estatal de una educación intercultural se anexa a las luchas por la defensa de sus territorios de la usurpación de colonos españoles, portugueses, holandeses e ingleses (SANTILLI, 1994). Para estos pueblos, el Estado mantiene un sistema de escuelas para indígenas provisto desde las políticas educativas. Son escuelas que están pensadas para entregar una educación que favorezca la integración de los indígenas a la sociedad brasilera, mediante la formación de "[...] trabajadores comprometidos con el progreso y con la patria, fortaleciendo el uso de los símbolos nacionales, el territorio, la religión, la lengua (portuguesa)" (REPETTO, 2012, p. 133), donde no se integran los conocimientos educativos indígenas al currículum escolar y tampoco la lengua.

En relación a las luchas indígenas, en materia de educación escolar, han sido favorecidas por la creación del Núcleo de Educación Indígena (NEI), fundado para administrar y supervisar las actividades que se desarrollaban en las escuelas situadas en comunidades indígenas (REPETTO, 2002). Sin embargo, las comunidades indígenas 
muestran su descontento con la educación que reciben sus hijos con el sistema educacional brasilero y con el desempeño de los profesores no indígenas, puesto que utilizan castigos violentos en las aulas y sobre valorizan la lengua portuguesa y cultura brasilera. Además, se hace notorio el desprecio por la cultura y la lengua indígena, y el carácter de la enseñanza basada en la aceptación cultural del dominador junto con la sumisión sociopolítica (REPETT0, 2012). Mientras tanto, las comunidades demandan una educación que brinde conocimientos útiles para sus contextos de vida.

Con respecto a las demandas de las comunidades, un grupo de investigadores y formadores crean la unidad administrativa y académica Instituto Insikiran de Formação Superior Indígena en la Universidad Federal de Roraima. Este instituto imparte un Curso de Formación de Profesores Intercultural, Bachillerato en Gestión Territorial Indígena y el Grado de Gestión en Salud Colectiva Indígena. Estas carreras universitarias han sido diseñadas y creadas para atender las demandas de los pueblos indígenas de Roraima (INSTITUTO..., 2016). Además, es preciso señalar que un grupo de investigadores y profesores del Instituto Insikiram trabajan en investigaciones y enseñanza desde el Método Inductivo Intercultural (GASCHÉ, 2008, 2010).

Desde la política normativa -Ley de Directrices y Bases para la Educación Brasilerase establece una enseñanza de la historia de Brasil construida desde una pluralidad epistemológica, tomando en cuenta las perspectivas indígenas, afrodescendientes y europea (REPETTO, 2002), es decir educación indígena en lenguaje portugués y propio. Se trata de una educación diferenciada para los pueblos indígenas, que respete y fomente sus culturas contextualizando los contenidos y haciendo partícipes a los miembros de las comunidades con prácticas pedagógicas desde una perspectiva intercultural bilingüe (BRASIL, 1996). Específicamente, el Referencial Curricular Nacional para as Escolas Indigenas (RCNEI) responde a la "[...] proposta de defender uma educação específica e diferenciada" (REPETTO, 2019, p. 83). La acción principal observada en la RCNEI es otorgar subsidios y orientaciones para que las escuelas elaboren programas de educación escolar para indígenas, que sean contextualizados a la realidad de sus comunidades, además de la creación de material didáctico y la formación de profesores (LACERDA; SOUZA; OLIVEIRA, 2017).

Sin embargo, a pesar de las buenas intenciones de la Ley, la práctica es diferente, puesto que no se dispone de material didáctico ni recursos económicos para llevar a cabo sus mandatos. Al respecto, Repetto argumenta que “[...] se puede entender la modalidad de educación escolar indígena como un subsistema del sistema nacional de educación, sobre el cual inciden esas otras modalidades, sin objetivos educativos claros" (2012, p. 139).

En este contexto, dentro de las comunidades indígenas hay dos posturas claras frente al tema educacional intercultural relacionado con las escuelas. Según los planteamientos de Repetto (2012), por una parte, un grupo respalda a los empresarios hacendados en territorios indígenas, haciéndose eco de su discurso en relación al desarrollo de la población, en mejorar la calidad de educación indígena e igualarla a la de la ciudad. Sin embargo, para los hacendados, el ámbito cultural, la episteme indígena y su lenguaje no tienen valor. Esta forma de pensar la educación intercultural representa el epistemicidio de los saberes y perspectivas indígenas (SANTOS, 2017). Por otra parte, está la postura de los 
indígenas que defienden sus costumbres, formas de vivir, aprender; asimismo, la retirada de los hacendados, la defensa de sus territorios, mediante cambios tomando en cuenta lo propio y construyendo una educación contextualizada y de calidad, contra argumentando el discurso de quienes dicen que la educación de la ciudad es mejor.

En relación a las dificultades que presenta la construcción de conocimientos desde una educación intercultural de la política normativa en Roraima, compartimos la visión general planteada por autores como Santos (2012, 2017); Tubino (2004, 2011) y Walsh (2010). En síntesis, estas visiones expresan las dificultades de plantear una educación intercultural solo para indígenas, puesto que perpetúa la colonización, silencia la discusión epistemológica, desvaloriza la cultura indígena y valoriza solamente lo occidental por sobre lo indígena. Esto plantea el desafío, urgente, de construir conocimientos interculturales basados en el respeto y el pluralismo epistemológico (OLIVÉ, 1999, 2004, 2009).

\section{Milpa educativa en México}

La Milpa educativa es un proyecto que se realiza en México, en los estados de Chiapas, Oaxaca, Puebla y Yucatán (NIGH; BERTELY, 2018). Este proyecto declara tener como objetivo la construcción de un modelo educativo intercultural plurilingüe, contextualizado y que ayude a las prácticas culturales de las comunidades indigenas (BERTELY; SARTORELLO; ARCOS, 2008). El sustento teórico es el método inductivo intercultural (MII) (GASCHÉ, 2008), definido como un método participativo que abarca la subjetividad reflexiva, la construcción cultural participativa, social y aprendizajes contextualizados. Asimismo, considera la información que aporta el estudiante desde sus experiencias de vida, con una estructura genérica que comienza desde la naturaleza, técnicas o transformación natural y fines sociales (GASCHÉ, 2010). El proyecto Milpas educativas surge por la necesidad de responder a los grupos indígenas por una educación contextualizada con sus formas de vida, que fomente el buen vivir y un espacio educativo que considere sus perspectivas de estrecha relación entre sociedad y naturaleza (SARTORELLO; AVILA, 2012).

En relación a este último punto, el proyecto de educación intercultural bilingüe tiene estrecha relación con la educación territorial (BERTELY, 2014) donde las Milpas educativas se pueden desarrollar en el contexto de la escuela o en casa de padres de familia, todo en pro de generar aprendizajes útiles para el buen vivir (BERTELY, 2014; NIGH; BERTELY, 2018).

Desde la premisa del buen vivir, los avances sociales y epistémicos que presenta este proyecto están relacionados con la consideración de los saberes indígenas y occidentales por medio del diálogo, para mejorar la construcción de conocimientos y fortalecimiento de la educación intercultural desde el análisis crítico de la realidad social y pluralidad epistémica (SARTORELLO, 2016).

En síntesis, las Milpas educativas son un proyecto educativo intercultural que tiene como sustento teórico-metodológico el MII (GASCHÉ, 2013), que ha ayudado a promover la resolución del problema de contextualización de la educación de los niños y niñas indígenas de los estados de Chiapas, Oaxaca, Puebla y Yucatán (NIGH; BERTELY, 2018). Desde la premisa y objetivo de alcanzar un buen vivir, según lo planteado por Sartorello y Avila (2012), los objetivos principales se pueden resumir en producción de 
conocimiento, valorización del territorio en razón indígena, educación para la autonomía de los pueblos indígenas, construcción de una educación que fomente el buen vivir y que combata el mal uso de la tierra (BERTELY; GASCHÉ; PODESTÁ, 2004). Sin embargo, este proyecto no es una política de los estados mexicanos donde se implementa, es una iniciativa de investigadores comprometidos con la construcción de una educación intercultural financiada por agencias privadas de desarrollo. Esta contribución es solo un inicio en este camino y aporte académico para demostrar que es posible la construcción de conocimientos desde una escuela intercultural con perspectiva indígena.

\section{Acción educativa kimeltuwün}

La acción educativa kimeltuwün es una propuesta teórica de investigadores sobre el conocimiento educativo mapuche desarrollado en la Araucanía, Chile. El kimeltuwün incluye también una propuesta metodológica descubierta en la educación familiar mapuche, que permite la construcción social de conocimientos educativos por parte de los padres de familias (QUILAQUE0; QUINTRIQUEO, 2017). Esta acción educativa demuestra que es posible llevar a cabo una educación propia mediante la interacción de un adulto con niños, niñas o jóvenes con el fin de aprender-enseñar contenidos relacionados con la familia, el territorio, respeto a las personas y autoridades tradicionales (QUILAQUE0; SARTORELLO, 2018).

El kimeltuwün se define sobre la base de seis etapas metodológicas: 1) gübam, es un concepto que representa el discurso actitudinal que permite a los aprendices valorar y fortalecer los conocimientos familiares y culturales; 2) güxam, es la conversación en el medio familiar para la socialización de saberes y conocimientos; 3) kimkimtun, hace relación a la formación bajo la metodología del aprender haciendo; 4) güneytun, consiste en aprender observando la naturaleza y las ceremonias realizadas en la comunidad; 5) günezuam, significa aprender a identificar los contenidos, valores y simbolismos buscando que los niños y niñas comprendan los contenidos conceptuales, procedimentales y actitudinales; 6) pepilün, es la aplicación de los conocimiento en las relaciones sociales y con la naturaleza. Estas etapas de la acción educativa tienen directa relación con la forma de ver el mundo del pueblo mapuche, que se caracteriza por su estrecha conexión y valoración de la naturaleza, por ende, los contenidos principales son la naturaleza, la persona, la familia, la comunidad, la espiritualidad y el territorio (QUILAQUE0; QUINTRIQUEO, 2017).

Sin embargo, la acción educativa mapuche kimeltuwün y episteme mapuche han estado ausentes en el ámbito escolar chileno, puesto que el ámbito normativo escolar promueve la universalidad, monoculturalidad eurocéntrica y una episteme exclusivamente occidental que se posiciona por sobre la mapuche. Así, el constante menosprecio de la episteme del conocimiento educativo mapuche en la mayoría de las relaciones interétnicas tiene como producto el racismo que se hace presente desde los primeros asaltos coloniales, pasando por la guerra Pacificación de La Araucanía, hasta nuestros días (RADOVICH; BALAZOTE, 2009). 
No obstante, en el ámbito normativo, la Ley Indígena n. 19.253 (CHILE, 1993) propone una serie de iniciativas tales como la preservación del lenguaje donde haya población indígena en una escuela o territorio y creación de un sistema de educación intercultural bilingüe, el objetivo propuesto es preparar a los indígenas para su desarrollo en su propia comunidad y en la sociedad global. Sin embargo, estos puntos no se condicen con la realidad, puesto que "[...] el conocimiento endógeno de los mapuches no es valorado, dentro de la reforma educativa chilena [...] con ello, la sociedad chilena tiende a estancarse en la idea de un mundo unicultural" (ROTHER, 2017, p. 81).

Por otra parte, se observa en la Araucanía el desarrollo del programa de educación intercultural bilingüe (PEIB) implementado por el Estado. Entre las críticas y reconocimientos está el replanteamiento de los asuntos indígenas en Chile, lo que ha aportado a la comprensión social de la diversidad y la extensión del tema de la interculturalidad al sistema público (WILLIAMSON, 2012). Sin embargo, las críticas se orientan a develar que el PEIB es una modalidad incluida en la educación especial y no se contempla en el espacio pedagógico regular de la escuela (WILLIAMSON, 2012), puesto que no considera el racismo, discriminación y la baja valoración social de los indígenas, al ser una educación diferenciada, pero en la que se mantienen las pruebas estandarizadas. Con esto se corre el riesgo de dificultar buenos resultados académicos, aumentar la frustración de estudiantes indigenas, entre otros problemas (RIEDEMANN, 2008).

\section{Semejanzas y diferencias de los tres casos con tensiones epistémicas}

Los tres casos analizados muestran semejanzas y diferencias en los planteamientos en relación al carácter y fondo de los estudios. Desde la episteme mapuche, en la acción educativa kimeltuwün, se observa el estudio en el que se describe, caracteriza, comprende y explica el modo en que las personas en contexto mapuche aprenden-enseñan en sus comunidades hace cientos de años, rescatando la episteme y tradición en el área educativa. A diferencia, la Milpa educativa es un proyecto didáctico que implementa un método educativo en contexto indígena. En este caso, se señala una serie de acciones propuestas desde los estudios interculturales previos a la implementación del proyecto, para proponer y ejecutar una educación de estudiantes indígenas en base al método inductivo intercultural.

Las investigaciones de Repetto y Carvalho (2015) se diferencian de las Milpas Educativas y la acción educativa kimeltuwün, debido a que su foco está mayormente en el área normativa de la educación ofrecida por el Estado, con un análisis crítico en relación a las comunidades indigenas donde ha investigado lo que viven los indigenas en materia educacional. Desde los estudios tratados en este artículo, se evidencia la episteme política en América Latina, la que es fundada desde los planteamientos occidentales, con el menosprecio de la visión indígena para llevar a cabo la implementación de la episteme occidental monoculturalizante (WALSH, 2010). Esto se refleja, por ejemplo, en las dificultades, conflictos y tensiones epistémicas de las comunidades indigenas que se suman a tensiones del sistema político normativo brasilero y los hacendados. 
Se observa, en general, que la educación en contextos interculturales en América Latina es de carácter funcionalista (ADAMS; KEE; LIN, 2001) en el que prima el factor técnico en "[...] los análisis de costo-beneficio, costo-eficiencia, costo-efectividad, la administración por objetivos, e indicadores sociales” (ESPINOSA, 2009). La episteme política funcionalista prima por sobre las políticas educacionales con enfoque crítico, que postulan que las legislaciones políticas deben ir en concordancia con la identificación y corrección de la represión, explotación, fuentes de dominación tomando un rol reivindicativo de los indígenas, grupos oprimidos y/o discriminados, mujeres o cualquiera que sufra una constante subyugación (PRUNTY, 1985).

A diferencia de la episteme educativa occidental, las epistemes educativas de los pueblos indígenas evidencian puntos en común que se originan de las luchas políticas en cada contexto, que data de la colonización europea de América (RADOVICH; BALAZOTE, 2009; RAMOS, 1992; SANTILLI, 1994). Este proceso histórico ha llevado a un epistemicidio del conocimiento indígena (RIVERA, 2010; SANTOS, 2012, 2017) y con ello un retraso de siglos en relación a la construcción de una educación con un enfoque intercultural fundada en el reconocimiento, diálogo y negociación (COHEN-EMERIQUE, 2013).

Las tres propuestas de las investigaciones revisadas comparten el pensamiento de la interculturalidad desde la relación de dominación colonizadora y sumisión indígena (GASCHÉ, 2008; RIVERA CUSICANQUI, 2010). Este reto lleva a las Milpas educativas a valorar y adoptar a la naturaleza como el laboratorio de aprendizaje. De igual forma, ocurre en el caso mapuche con el kimkimtun o aprender haciendo, el güneytun aprender observando y el pepilün aplicación del conocimiento en la naturaleza.

En relación a las dificultades que presenta la educación intercultural en los tres casos analizados, compartimos la visión general de la literatura revisada en este ensayo (REPETTO, 2012; RIVERA CUSICANQUI, 2010; SANTOS, 2012, 2017; TUBINO, 2004, 2011; WALSH, 2010), puesto que se presentan alternativas para establecer una educación intercultural contextualizada a la historia de colonización de cada país y la implementación de un sistema educativo intercultural plurilingüe. Sin embargo, observamos que la visión funcionalista de la política en la mayor parte de los países de América Latina perpetúa la colonización, silencia la discusión epistemológica, desvaloriza la cultura indígena y exacerba la valorización de lo occidental, dificultando el avance hacia una educación intercultural en las escuelas basadas en el respeto y el pluralismo epistemológico (OLIVÉ, 1999, 2004, 2009).

\section{Reflexiones finales}

Los planteamientos y reflexiones de este artículo son homologables con la realidad que viven estudiantes de escuelas situadas en todos los contextos indígenas, particularmente en experiencias de educación intercultural dirigidas exclusivamente a pueblos indígenas de América Latina. Por ejemplo, Perú (ESPINOSA, 2014) y Ecuador (SCHRODER, 2006), donde se observa un escaso accionar de los profesores en la toma de conciencia de la pluralidad cultural de sus salas de clase (SINGH, 1988) y como consecuencia subestiman las epistemes indígenas en la construcción de conocimientos en 
las escuelas. Consecuentemente, una construcción de educación desde solo una episteme repercute tanto en lo sistémicoestructural de la educación, como en lo micro. Por ejemplo, ello se observa en las dificultades del aprendizaje de matemática por parte de los indígenas producto del rechazo de sus epistemes (GONZALEZ, 2018).

Desde las investigaciones analizadas, planteamos una construcción de conocimientos que tome en cuenta las epistemes indígenas, sin el desprecio por los saberes del dominado y mediado por diálogos horizontales que superen las asimetrías de poder. Diálogos interculturales que reconozcan la historia del epistemicidio de la colonización que continúan provocando los Estados nacionales en el tiempo (SANTOS, 2017). En resonancia con los planteamientos de Fornet-Betancourt (1994), compartimos que "[...] la necesidad del diálogo intercultural en América Latina está conectada con la historia de la conquista y de la colonización del subcontinente" (FORNET-BETANCOURT, 1994, p. 5).

La necesidad de avanzar en la construcción de conocimientos interculturales en las escuelas se hace cada vez más necesaria. Por ello, es fundamental analizar críticamente los estudios realizados en América Latina, con el fin de avanzar en la construcción de una educación intercultural participativa. Educación en que no se excluya ninguna episteme, se valoricen todas por igual y se fomente el diálogo entre personas que viven en un mismo contexto, para construir una educación con enfoque intercultural donde todos aprendemos de todos, sin elevar un conocimiento por sobre otro.

Constatamos que, en general los planteamientos de la educación intercultural es una herramienta para el reconocimiento cultural de los niños indígenas; sin embargo, los indígenas manifiestan que sus efectos son de segregación social al no considerar la población no indígena. Por esta razón, se propone una educación intercultural para todos y no solo para los indígenas, puesto que la segregación en la educación contribuye a la perpetuación de las relaciones asimétricas. Así, desde el pluralismo epistemológico nos preguntamos: ¿crear una educación intercultural para indígenas es la mejor opción? o ¿una educación intercultural para todos ya sea mestizo, migrante, indígena, de contexto rural o urbano? ¿Será mejor que las adecuaciones educacionales se hagan en relación al contexto, territorio, sociedad, cultura, y no solamente en razón de la decisión política funcionalista del Estado?

En conclusión, la construcción de conocimientos en base a un diálogo de saberes educativos según la evidencia empírica y teórica enriquece a las naciones. Planteamos que el diálogo de saberes educativos sea basado en la construcción de conocimientos producidos tanto en las escuelas como en las familias de los pueblos indígenas. Es decir, sustentada en la pluralidad epistemológica, donde el Estado no sea el único quien decida qué y cómo aprender, puesto que las personas en contexto indígena conocen el sistema educacional occidental y su propio sistema o forma de aprender.

\section{Referencias}

ADAMS, Don; KEE, Geok Hwa; LIN, Lin. Linking research, policy, and strategic planning to education development in Lao People's Democratic Republic. Comparative Education Review, Chicago, v. 45, n. 2 , p. 220-241, 2001. 
ALCOREZA, Raúl Prada. Epistemología pluralista. In: ZAMBRANA, Amílcar B. (ed.). Pluralismo epistemológico: reflexiones sobre la educación superior en el Estado Plurinacional de Bolívia. Cochabamba: Funproeib, 2014. p. 13-54.

ASSIES, Willem. Los pueblos indígenas, la tierra, el territorio y la autonomía en tiempos de globalización. In: MARTÍ I PUIG, Salvador (ed.). Pueblos indígenas y política en América Latina. Barcelona: Cidob, 2007. p. 227-246.

BANKS, James. Multicultural education and curriculum transformation. Journal of Negro Education, Cambridge, v. 64, n. 4, p. 390-400, 1995.

BERGER, Peter; LUCKMANN, Thomas. The social construction of reality: a treatise in the sociology of knowledge. London: Penguin Books, 1991.

BERTELY, María. Educación intercultural, alfabetización territorial y derechos indígenas en y desde Chiapas. Artículos y Ensayos de Sociología Rural, Texcoco, v. 9, n. 17, p. 23-39, 2014.

BERTELY, María. Enfoques postcoloniales y movimiento político y pedagógico intercultural en y desde Chiapas, México. Relaciones, Michoacán, v. 36, n. 141, p. 75-102, 2015.

BERTELY, María; GASCHÉ, Jorge; PODESTÁ, Rossana. Educando en la diversidad: investigaciones y experiencias educativas interculturales y bilingües. Quito: Abya-Yala, 2004.

BERTELY, María; SARTORELLO, Stefano Claudio; ARCOS, Francisco. Vigilancia, cuidado y control étnicopolítico: red de educación inductiva intercultural. Quito: Abya-Yala, 2008.

BRASIL. Lei n 9394. Lei de diretrizes e bases da educação nacional. Brasília, DF: Senado Federal, 1996.

BRIONES, Claudia. Conocimientos sociales, conocimientos académicos: asimetrías, colaboraciones, autonomías. DesiguALdades.net Working Paper Series, Berlín, n. 39, p. 1-27, 2013.

CHILE. Ministerio de Planificación y Cooperación. Ley $n^{\circ} 19253$ de 5 de octubre de 1993. Establece normas sobre protección, foment y Desarrollo de los indígenas, y crea la Cooperación Nacional de Desarrollo Indígena. Santiago de Chile: Biblioteca del Congreso Nacional, 1993. Disponible en: https://www.bcn.cl/ leychile/navegar?idNorma=30620. Acceso en: 20 jul. 2019.

COHEN-EMERIQUE, Margalit. Per un enfocament intercultural en la intervenció social. Educación Social, Barcelona, n. 54, p. 11-38, 2013.

COURTIS, Christian. Apuntes sobre la aplicación del Convenio 169 de la oit sobre pueblos indígenas por los Tribunales de América Latina. Revista Internacional sobre Derechos Humanos, São Paulo, v. 6, n. 10, p. 53-78, 2009.

DONOSO, Sebastián. Chile y el convenio 169 de la OIT: reflexiones sobre un desencuentro. Temas de la Agenda Pública, Santiago de Chile, v. 3, n. 16, p. 1-13, 2008. Disponible en: http://politicaspublicas.uc.cl/ wp-content/uploads/2015/02/chile-y-el-convenio-169-de-laoit-reflexiones-sobre-un-desencuentro.pdf. Acceso en: 20 jul. 2019. 
ESPINOSA, Oscar. Los planes de vida y la política indígena en la Amazonía peruana. Anthropologica, Lima, v. 32, n. 32, p. 87-114, 2014.

ESPINOSA, Oscar. Reflexiones sobre los conceptos de "política", políticas públicas y política educacional. Education Policy Analysis Archives/Archivos Analíticos de Políticas Educativas, Phoenix, v. 17, n. 8, p. 1-13, 2009.

FERRÃO CANDAU, Vera María. Educación intercultural en América Latina: distintas concepciones y tensiones actuales. Estudios pedagógicos, Valdivia, v. 36, n. 2, p. 333-342, 2010.

FORNET-BETANCOURT, Raúl. Filosofía intercultural. México, DC: Universidad Pontificia de México, 1994.

FORNET-BETANCOURT, Raúl. Lo intercultural: el problema de su definición. Pasos, Madrid, v. 103, p. 1-3, 2002.

GASCHÉ, Jorge. Éxitos y fracasos de una propuesta educativa basada sobre el "Método Inductivo Intercultural" en implementada en el Perú, México y el Brasil. Isees, Santiago de Chile, n. 13, p. 17-34, 2013.

GASCHÉ, Jorge. La motivación política de la educación intercultural indígena y sus exigencias pedagógicas. ¿Hasta dónde abarca la interculturalidad? In: BERTELY, María; GASCHÉ, Jorge; PODESTÁ, Rossana. Educando en la diversidad. Quito: Abya-Yala, 2008. p. 367-397.

GASCHÉ, Jorge. ¿Qué son "saberes" o "conocimientos" indígenas, y qué hay que entender por "diálogo"? In: PÉREZ, Catalina; ECHEVERRI, Juan (ed.). Memorias: primer encuentro amazónico de experiencias de diálogo de saberes. Leticia: Universidad Nacional de Colombia, 2010. p. 17-31.

GONZALEZ, Fredy Enrique. Historia de la educación matemática en Latinoamérica: 10 claves para su comprensión. Unión, São Paulo, n. 52, p. 279-305, 2018.

GROSFOGUEL, Ramón. Del «extravismo económico» al «extravismo epistémico» y al «extravismo ontológico»: una forma destructiva de conocer, ser y estar en el mundo. Tabula Rasa, Bogotá, n. 24, p. 123-143, 2016.

GUILHERME, Manuela; DIETZ, Gunther. Difference in diversity: multiple perspectives on multicultural, intercultural, and transcultural conceptual complexities. Journal of Multicultural Discourses, London, v. 10, n. 1, p. 1-21, 2015.

INSTITUTO INSIKIRAN DE FORMAÇÃO SUPERIOR INDÍGENA. Apresentação. Boa Vista: UFRR, 2016. Disponible en: http://ufrr.br/insikiran/index.php?option=com_content\&view=article\&id=60\&lte. Acceso en: 2 my. 2020.

LACERDA, Neomar; SOUZA, Maria Elizabete; OLIVEIRA, Rachel. A matemática no currículo das escolas indígenas: um desafio da educação matemática. Revista Latinoamericana de Etnomatemática, San Juan de Pasto, v. 10, n. 3, p. 149-166, 2017. 
LÓPEZ, Luis Enrique; KÜPER, Wolfgang. La educación intercultural bilingüe en América Latina: balance y perspectivas. Revista Iberoamericana de educación, Madrid, v. 20, p. 17-85, 1999.

MANDULÃO, Giovana et al. Projeto Vidas Paralelas Indígena: revelando os povos Macuxi e Wapixana de Roraima, Brasil. Tempus Actas de Saúde Coletiva, Brasilia, DF, v. 6, n. 1, p. 63-70, 2012.

NIGH, Ronald; BERTELY, Maria. Conocimiento y educación indígena en Chiapas, México: un método intercultural. Diálogos Sobre Educación, Zapopan, v. 9, n. 16, 2018.

OLIVÉ, León. La exclusión del conocimiento como violencia intercultural. Polylog, Viena, v. 5, p. 1-14, 2004.

OLIVÉ, León. Multiculturalismo y pluralismo. México, DC: Paidós, 1999.

OLIVÉ, León. Por una auténtica interculturalidad basada en el reconocimiento de la pluralidad epistemológica. In: OLIVÉ, León; SOUZA SANTOS, Boaventura de; SALAZAR, Cecilia. Pluralismo epistemológico. La Paz: Clacso, 2009. p. 19-30.

OIT. Organización Internacional del Trabajo. Convenio sobre pueblos indígenas y tribales. Genebra: OIT, 1989. Disponible en: <http://www.ilo.org/dyn/normlex/es/f?p=NORMLEXPUB:12100:0::NO::P12100_ ILO_CODE:C169>. Acceso en: 20 jul. 2019.

PRUNTY, John. Signposts for a critical educational policy analysis. Australian Journal of Education, Melbourne, v. 29, n. 2, p. 133-140, 1985.

QUILAQUEO, Daniel; QUINTRIQUEO, Segundo. Métodos educativos mapuche: retos de la doble racionalidad educativa. Aportes para un enfoque de educación intercultural. Temuco: Universidad Católica de Temuco, 2017.

QUILAQUEO, Daniel; SARTORELLO, Stefano. Retos epistemológicos de la interculturalidad en contexto indígena. Alpha, Osorno, n. 47, p. 47-61, 2018.

RADOVICH, Juan Carlos; BALAZOTE, Alejandro. El pueblo mapuche contra la discriminación y el etnocidio. In: GHIOLDI, Gerardo (comp.). Historia de las familias mapuche Lof Paichil Antriao y Lof Quintriqueo de la margen Norte del lago Nahuel Huapi. Archivos del Sur-Villa La Angostura. Córdoba: Ferreyra, 2009. p. 86-98.

RAMOS, María Eugenia Reyes. El reparto de tierras y la política agraria en Chiapas, 1914-1988. Coyoacán: UNAM, 1992.

REPETTO, Maxim. Educación escolar indígena en Roraima, Amazonas brasileño: del conflicto intercultural a la construcción de propuestas educativas. Inter-American Journal of Education for Democracy, Indiana, v. 4, n. 1, p. 21-21, 2012.

REPETTO, Maxim. O conceito de interculturalidade: trajetórias e conflitos desde América Latina. Textos e Debates, Boa Vista, v. 2, n, 33, p. 69-88, 2019. 
REPETTO, Maxim. Reivindicaciones de educación indígena en Roraima-Brasil. Revista de la Academia, Santiago de Chile, n. 7, p. 145-160, 2002.

REPETTO, Maxim; CARVALHO, Fabiola. Experiencias de investigación educativa intercultural en la formación de maestros indígenas en Roraima, Brasil. Desacatos, México DC, n. 48, p. 50-65, 2015.

RIEDEMANN, Andrea. La educación intercultural bilingüe en Chile:¿ampliación de oportunidades para alumnos indígenas? Indiana, Berlín, v. 25, p. 169-193, 2008.

RIVERA CUSICANQUI, Silvia. Ch'ixinakax utxiwa: una reflexión sobre prácticas y discursos descolonizadores. Buenos Aires: Tinta Limon, 2010.

ROMERO, Carlos Giménez. Pluralismo, multiculturalismo e interculturalidad. Educación y Futuro, Madrid, n. 8, p. 11-20, 2003.

ROTHER, Tanja. Conflicto intercultural y educación en Chile: desafíos y problemas de la educación intercultural bilingüe (EIB) para el pueblo mapuche. Revista Austral de Ciencias Sociales, Valdivia, n. 9, p. 71-84, 2017.---

SANTILLI, Paulo. Fronteiras da República: história e política entre os Macuxi no Vale do Rio Branco. São Paulo: NHII-USP: FAPESP, 1994.

SANTOS, Boaventura de Sousa. Cuando los excluidos tienen derecho: justicia indígena, plurinacionalidad e interculturalidad. In: SANTOS, Boaventura de Sousa; GRIJALVA, Agustín. Justicia indígena, plurinacionalidad e interculturalidad en Ecuador. Quito: Fundación Rosa Luxemburg, 2012. p. 13-50.

SANTOS, Boaventura de Sousa. Justicia entre saberes: epistemologías del sur contra el epistemicidio. Madrid: Morata, 2017.

SARTORELLO, Stefano Claudio. Política, epistemología y pedagogía: el método inductivo intercultural en una escuela tseltal de Chiapas, México. LiminaR, Chiapas, v. 14, n. 1, p. 121-143, 2016.

SARTORELLO, Stefano Claudio; AVILA, Leon Enrique. El buen vivir: miradas desde adentro de Chiapas. Chiapas: Unich, 2012.

SCHMELKES, Sylvia. La educación intercultural: un campo en proceso de consolidación. Revista Mexicana de Investigación Educativa, México, DC, v. 9, n. 20, p. 9-13, 2004.

SCHRODER, Barbara. Native science, intercultural education and place-conscious education: an Ecuadorian example. Educational Studies, London, v. 32, n. 3, p. 307-317, 2006.

SINGH, B. R. Neutrality and education in a pluralist society. Educational Studies, London, v. 14, n. 2, p. 121-138, 1988.

SLEETER, Christine E. Multicultural education past, present, and future: Struggles for dialog and powersharing. International Journal of Multicultural Education, St. Davids, v. 20, n. 1, p. 5-20, 2018. 
TUBINO, Fidel. Del interculturalismo funcional al interculturalismo crítico. Rostros y Fronteras de la Identidad, Lima, v. 158, p. 1-9, 2004.

TUBINO, Fidel. El nivel epistémico de los conflictos interculturales. Revista Electrónica Construyendo Nuestra Interculturalidad, Lima, v. 7, n. 6-7, p. 1-14, 2011.

WALSH, Catherine. Interculturalidad crítica y educación intercultural. Construyendo Interculturalidad Crítica, La Paz, v. 75, p. 75-96, 2010.

WILLIAMSON, Guillermo. ¿Educación multicultural, educación intercultural bilingüe, educación indígena 0 educación intercultural? Cuadernos Interculturales, Viña del Mar, v. 2, n. 3, p. 23-34, 2004.

WILLIAMSON, Guillermo. Institucionalización de la educación intercultural bilingüe en Chile: Notas y observaciones críticas. Perfiles Educativos, México, DC, v. 34, n. 138, p. 126-147, 2012.

Recibido en: 05.12.2019

Revisado en: 28.04.2020

Aprobado en: 01.09.2020

Esteban Guillermo Saavedra Vallejos es estudiante de doctorado en Educación en consorcio de la Universidad Católica de Temuco, Chile (UCT). Máster en Dirección y Gestión de la Actividad Física y Deporte, Universitat de València, España.

Daniel René Quilaqueo Rapimán es doctor en Sociología, Université de la Sorbonne Nouvelle, Francia. Director del doctorado en Educación de la Universidad Católica de Temuco, Chile (UCT). Es investigador asociado del Centre Interuniversitaire d'Études et de Recherches Autochtones, I'Université Laval, Canadá. 